\title{
Necrotizing Fasciitis: Risk Factors and Outcomes in the COVID-19 Era
}

\author{
Rohit Chauhan ${ }^{1}$, Devadatta Poddar $^{2}$, Prateek Lohchab $^{3}$ \\ ${ }^{1,3}$ Post Graduate Resident, Department of General Surgery, ABVIMS \& Dr RML Hospital, New Delhi \\ ${ }^{2}$ Associate Professor, Department of General Surgery, ABVIMS \& Dr RML Hospital, New Delhi \\ Corresponding Author: Devadatta Poddar
}

\begin{abstract}
Background: Necrotizing Fasciitis is a severe, rapidly spreading soft tissue infection with high morbidity and mortality. Diabetes mellitus, hypertension, and immune suppression are the some of the predisposing factors for the disease. The objective of this study was to study the risk factors and outcomes of necrotizing fasciitis in the COVID-19 era.
\end{abstract}

Methods and Materials: a retrospective analysis of the data of forty-four patients admitted with the diagnosis of NF from April 2020 to April 2021was done at Dr Ram Manohar Lohia Hospital, New Delhi. Data was recorded in MS Excel spreadsheet program. SPSS v23 (IBM Corp.) was used for data analysis. Descriptive statistics were elaborated in the form of means/standard deviations and medians/IQRs for continuous variables, and frequencies and percentages for categorical variables. Chi-square test $\left(X^{2}\right)$ was used for group comparisons of categorical data.

Results: Out of forty-four patients, 26 (59.10\%) were male and $18(40.9 \%)$ were female. The mean age was 54.2 years (+ 14.4 years). Extremities $(68.2 \%)$ were the most commonly involved site followed by perineum $(29.5 \%)$ and abdominal wall (2.2\%). Diabetes mellitus (DM) was the most prevalent comorbidity. 10 patients had both DM and hypertension (HTN). Fourteen patients $(32 \%)$ had a polymicrobial infection on tissue culture. Rest thirty patients had involvement of gram positive (44\%) and gram negative (24\%) organisms. Twenty-two patients $(50 \%)$ had healthy granulation tissue as the final outcome. However, the rest $50 \%$ patients had a poor outcome in form of amputation (13.63\%) and mortality $(36.36 \%)$. Average duration from onset of symptoms to presentation at the hospital was $7.11(+3.47)$ days. Patients who presented early to the hospital within 7 days of symptom onset had a good outcome in the form of healthy granulation tissue $\left(X^{2}=7.62\right.$ and $\mathrm{p}$ $<0.05$ ).

Conclusions: Early diagnosis and prompt surgical intervention in necrotizing fasciitis is the key to its management. Delayed presentation was because of restricted movement, government-imposed lockdowns, patients ignoring their symptoms, and resorting to selfmedications. The delay in diagnosis leading to a delay in treatment is one of the major factors contributing to poor outcomes in the COVID-19 era.

Keywords: necrotizing fasciitis, risk factors, COVID 19

\section{INTRODUCTION}

Necrotizing Fasciitis (NF) is an aggressive soft tissue infection associated with a high mortality rate. Extremities, perineum, and abdominal wall are some of the commonly involved areas. Rarely, other sites such as head, neck, or breast can also be involved ${ }^{[1]}$. Patients with co-morbidities such as diabetes mellitus, hypertension, and those on immunosuppressive drugs are at high risk of developing this condition [Error! Reference source not found.,2]. Early diagnosis and intervention are the key to its management. A delay in presentation can have undesirable outcomes such as multiple debridement, prolonged hospital stay, need for grafting, amputation, and even death ${ }^{[1}$ 4]. 
Since March 2020, the COVID-19 pandemic had majorly started to affect India and a nationwide lockdown was announced on 23 March, 2020. All non-essential services including elective surgeries were cancelled and people were ordered to stay at home in order to limit the virus from spreading. The fear of contracting COVID19 forced people to avoid seeking healthcare services on time leading to a delay of presentation, diagnosis, treatment, and a related increase in the morbidity and mortality due to $\mathrm{NF}^{[5]}$. We aim to see the average duration after which the patient reported to the hospital after onset of symptoms and its consequences on the outcomes. Judicious use of telemedicine could be of help to these patients in the COVID-19 era. Patient awareness can be increased through telemedicine and also direct them to proper medical care at the earliest possible time after symptom onset.

\section{MATERIALS AND METHODS}

Forty-four patients admitted with a diagnosis of NF at Dr Ram Manohar Lohia Hospital, New Delhi during the period of April 2020 to April 2021 were included in the study. To avoid confounding by the coronavirus as a cause of mortality, patients with a positive COVID-19 report were excluded from the study. Patients positive for HIV and patients with a history of trauma were also excluded from the study. The patient were diagnosed on the basis of clinical findings such as characteristic skin changes, fever, pain out of proportion to physical findings, leucocytosis, and histopathological findings suggestive of NF. The data of these patients was retrospectively analysed. The data included the age, gender, time from onset of symptoms to presentation, co-morbidities, and causative microorganisms. Laboratory parameters such as haemoglobin, serum sodium, creatinine, and leukocyte count at presentation were noted. Site of involvement such as extremities, perineum, and abdominal wall were recorded. Initially all patients were treated with empirical antibiotics using ceftriaxone, amikacin, and metronidazole. Antibiotic was changed after forty-eight hours based on culture and sensitivity. Wound inspection and serial debridement were done daily till no further spread was seen and healthy granulation was noticed. The outcomes of the disease at the end of seven days were healthy granulation tissue, amputation, and mortality. SPSS v23 (IBM Corp.) was used for data analysis. Descriptive statistics were elaborated in the form of means/standard deviations and medians/IQRs for continuous variables, and frequencies and percentages for categorical variables. Chi-square test was used for group comparisons of categorical data.

\section{RESULTS}

Out of forty-four patients, 26 $(59.10 \%)$ were male and $18(40.9 \%)$ were female. The mean age (Standard Deviation) was 54.2 years $(+14.4$ years).Extremities $(68.2 \%)$ were the most commonly involved site followed by perineum $(29.5 \%)$ and abdominal wall $(2.2 \%)$. Table 1 shows the number of patients and the site involved by NF. In patients with extremity as site of NF, 9 had mortality, 6 underwent amputation, and rest 15 had healthy granulation tissue as an outcome. Those with perineum as site of $\mathrm{NF}$, mortality as an outcome were seen in 7 patients, while 6 patients had a healthy granulation tissue. The only patient with abdominal wall as site of NF had mortality as outcome.

Table 1: Distribution of patients according to the site of NF

\begin{tabular}{|l|l|}
\hline Site & Number of patients $(\mathbf{n}=\mathbf{4 4})$ \\
\hline Extremities & $30(68.18 \%)$ \\
\hline Perineum & $13(29.54 \%)$ \\
\hline Abdominal Wall & $1(2.27 \%)$ \\
\hline
\end{tabular}

The distribution of patients according to their comorbidity is depicted in Table 2. Diabetes mellitus (DM) was the most prevalent comorbidity. Ten patients had both DM and hypertension (HTN). Eleven patients $(25 \%)$ had no comorbidity. Out of the 33 patients with comorbidity, 20 had a poor outcome in the form of amputation $(n=5)$ and mortality $(n=15)$. In 
those without comorbidity, there was a single case each of amputation and mortality.

Table 2: Distribution of patients according to their comorbidity

\begin{tabular}{|l|l|}
\hline Comorbidity & Number of Patients $(\mathbf{n}=\mathbf{4 4})$ \\
\hline Diabetes Mellitus & $21(47.72 \%)$ \\
\hline Hypertension & $2(4.54 \%)$ \\
\hline Both DM \& HTN & $10(22.72 \%)$ \\
\hline None & $11(25 \%)$ \\
\hline
\end{tabular}

14 patients $(32 \%)$ had a polymicrobial infection on tissue culture. Rest of the patients had involvement of gram positive (44\%) and gram negative (24\%) organisms. The distribution of patients according to the organism involved is shown in Table 3.

Table 3: Distribution of patients according to the organism involved

\begin{tabular}{|l|l|}
\hline Microorganism & Number of patients $(\mathbf{n}=\mathbf{4 4})$ \\
\hline Polymicrobial & $14(32 \%)$ \\
\hline Gram Positive & $19(43 \%)$ \\
\hline Gram Negative & $11(25 \%)$ \\
\hline
\end{tabular}

Average duration from onset of symptoms to presentation at the hospital was $7.11(+3.47)$ days. The outcomes at the end of course of treatment were healthy granulation tissue, amputation, and mortality. The distribution of patients according to their outcomes and the average duration (in days) of presentation to hospital from onset of symptoms is depicted in Table 4. Patients with a longer duration of symptoms onset to presentation had a worse outcome in the form of amputation and mortality.22 patients (50\%) had healthy granulation tissue as the final outcome. However, the rest $50 \%$ patients had a poor outcome in form of amputation (13.63\%) and mortality (36.36\%).

Table 4: Distribution of patients according to the outcomes and time of presentation

\begin{tabular}{|l|l|l|}
\hline Outcomes & $\begin{array}{l}\text { Number of } \\
\text { patients }\end{array}$ & $\begin{array}{l}\text { Symptoms onset } \\
\text { to presentation } \\
\text { (in days) }\end{array}$ \\
\hline Healthy granulation tissue & $22(50 \%)$ & $5.82(+3.49)$ days \\
\hline Amputation & $6(13.63 \%)$ & $7.50(+2.81)$ days \\
\hline Mortality & $16(36.36 \%)$ & $8.75(+3.08)$ days \\
\hline
\end{tabular}

Patients were divided into those who presented before and after the average duration of presentation and a group comparison were done for their outcomes using the chi-square test. Similar group comparison was done for those with and without a comorbidity, site of NF, and type of microorganism on culture). Table 5 depicts the degree of association between various factors (the site of occurrence of $\mathrm{NF}$, comorbidities, duration from symptoms onset to presentation) and the outcomes. The chi-square test $\left(X^{2}\right)$ showed that incidence of poor outcome in the form of amputation and mortality was more in patients who had a comorbidity and also in those who presented late (>7 days) after onset of symptoms. However, no such correlation could be drawn between the outcome and the site of occurrence and type of microorganism on culture.

Table 5: chi-square statistic and p-value for various factors against the outcomes

\begin{tabular}{|c|c|c|c|c|}
\hline & Site & Comorbidity & Symptoms onset to presentation & Microorganism on culture \\
\hline $\begin{array}{l}\text { Outcomes } \\
\text { (Mortality/Amputation/ } \\
\text { Healthy granulation tissue) }\end{array}$ & $\begin{array}{l}X^{2}=1.891 \\
p=.3880\end{array}$ & $\begin{array}{l}X^{2}=7.572 \\
p=.0226\end{array}$ & $\begin{array}{l}X^{2}=7.626 \\
p=.0220\end{array}$ & $\begin{array}{l}X^{2}=0.933 \\
\mathrm{p}=.9196\end{array}$ \\
\hline
\end{tabular}

\section{DISCUSSION}

$\mathrm{NF}$ is an aggressive disease and is associated with a high morbidity and mortality. The infection is rapidly progressive involving the skin, subcutaneous tissue, and the fascia. Prompt intervention in the form of serial surgical debridement is crucial. A delay in the presentation, diagnosis, or intervention can lead to adverse outcomes such as amputation and mortality ${ }^{[1-4]}$. LRINEC score is a scoring system to assess the severity of necrotizing fasciitis. However, the LRINEC score does not take into account the time from onset of symptoms to presentation at the hospital ${ }^{[6]}$.

In this study, $59.10 \%$ patients were male and $40.90 \%$ were female. 54.2 years was the mean age at presentation. Extremities were the most commonly involved site (Table 1). Of all the patients presenting with NF, $32 \%$ had a polymicrobial infection. Those with monomicrobial infection, $43 \%$ patients had 
a gram-positive organism and $25 \%$ had a gram-negative organism in their culture (Table 3). These results are similar to those in the studies done by Nissar Shaikh et al (2015) and Gaby Jabbour et al (2016) ${ }^{[1, \text { Error! }}$ Reference source not found.]. However, the studies did not comment upon the relationship of these factors with the outcome. In our study, the site of occurrence and the type of microorganism grown in culture had no significant correlation with the outcomes (p>0.05) (Table 5).

Diabetes mellitus was the most frequently associated comorbidity followed by hypertension in our study on patients with NF (Table 2). In the studies done by Nissar Shaikh (2015), PatcharinKhamnuan et al (2015), Arif N et al (2016), andGaby Jabbour et al (2016), it is established that comorbidities such as diabetes mellitus, hypertension, immune-suppression, and chronic renal failure are associated with NF. These comorbidities are also associated with a high mortality ${ }^{[1-2,7]}$. In our study, patients who had a comorbidity had poor outcome in the form of amputation or mortality $\left(X^{2}\right.$ $=7.572$ and $\mathrm{p}=.0226$ ) (Table 5). Management of diabetics and hypertensive patients were most affected after the emergence of COVID-19 due to a delay in diagnosis of these conditions. Patients previously diagnosed with the disease were also affected due to lack of essential medicines, insufficient staff, and utilization of most services of health care workers for COVID-19 patients ${ }^{[8]}$. There is a delay in diagnosis of comorbidities as well as inadequate monitoring and treatment of previously diagnosed patients.

The mean duration from the onset of symptoms to presentation at hospital was 7.11 (+3.47) days (Table 4). This was more than that in the study by Nissar Shaikh et al (2015) in the pre COVID-19 era where the average duration of symptoms was 4 days [Error! Reference source not found.]. Inour study the mortality rate was $36.36 \%$ which is significantly higher than that reported in the pre COVID-19 era ${ }^{[1,2]}$. Most of the deaths were seen in those patients who had co morbidity in the form of diabetes mellitus. However, increased mortality was also seen in patients with delayed presentation to the hospital. Leslie Kobayashi et al (2011) and Gaby Jabbour et al (2016) in their study have found that a delay in presentation, diagnosis, or intervention is associated with high mortality reaching up to $44 \%{ }^{[1,4]}$. YC Lee et al (2014) in their study concluded that a delayed treatment beyond 3 days post symptom onset was associated with a significantly higher mortality and is an independent factor predicting poor prognosis ${ }^{[9]}$. In this study, patients with amputation and mortality as an outcome presented after an average duration of 7.50 $(+2.81)$ days and $8.75(+3.08)$ days post symptom onset, respectively. A significant correlation was present between a delay in presentation and the outcomes $\left(X^{2}=7.626\right.$ and $\mathrm{p}=.0220) \quad$ (Table 5). Government imposed lockdown hindering access to healthcare, lack of public transport services due to lockdown, abolition of elective care, and cessation of various screening programmes were some of the major factors for the delay in presentation besides the fear of contracting COVID-19 at healthcare centres ${ }^{[8]}$.

The disruption in access to health care services in the COVID-19 era accompanied by various other factors such as lack of physical activity, mental stress, unhealthy diet, and use of alcohol have led to an increase in the complications due to NF. The various restrictions in movement adopted by the government are particularly problematic for those living in rural or remote areas and those requiring regular care. A plan to mitigate these factors by the use of telemedicine or building alternate facilities is necessary to ensure continued services. Prompt diagnosis and early referral to seek medical care is necessary to prevent morbidity and mortality of such patients ${ }^{[8]}$.

\section{CONCLUSION}

$\mathrm{NF}$ is an aggressive disease associated with high morbidity and mortality requiring prompt diagnosis and 
intervention. Patients with comorbidity such as diabetes and hypertension are particularly vulnerable to the disease. A delay in presentation, diagnosis, or treatment is associated with higher mortality. The study has highlighted that even though the risk factors are similar to the pre COVID-19 era, the outcomes differ in the form of increased morbidity and mortality in the COVID-19 era. There is an urgent need to mitigate these factors to prevent morbidity and mortality due to NF.

\section{Acknowledgement: None}

\section{Conflict of Interest: None}

\section{Source of Funding: None}

\section{Ethical Approval: Approved}

\section{REFERENCES}

1. Jabbour G, El-Menyar A, Peralta R, Shaikh $\mathrm{N}$, Abdelrahman $\mathrm{H}$, Mudali IN et al. Pattern and predictors of mortality in necrotizing fasciitis patients in a single tertiary hospital. World J Emerg Surg. 2016; 11:40. Published 2016 Aug 8. doi:10.1186/s13017016-0097-y

2. Shaikh N, El-Menyar A, Mudali IN, Tabeb A, Al-Thani H. Clinical presentations and outcomes of necrotizing fasciitis in males and females over a 13-year period. Ann Med Surg. 2015;4 :355-60. https://doi.org/10.1016/j.amsu.2015.09.005

3. Khamnuan $P$, Chongruksut W, Jearwattanakanok K, Patumanond J, Yodluangfun S, Tantraworasin A. Necrotizing fasciitis: risk factors of mortality. Risk ManagHealthc Policy. 2015;8 :1-7. doi: 10.2147/RMHP.S77691.eCollection 2015.
4. Kobayashi L, Konstantinidis A, Shackelford $\mathrm{S}$, Chan LS, Talving $\mathrm{P}$, Inaba $\mathrm{K}$ et al. Necrotizing soft tissue infections: delayed surgical treatment is associated with increased number of surgical debridements and morbidity. J Trauma. 2011; 71(5):14005 .

5. Masroor S. Collateral damage of COVID-19 pandemic: delayed medical care. J Card Surg 2020;35(6):1345-1347

6. Hsiao CT, Chang CP, Huang TY, Chen YC, Fann WC. Prospective Validation of the Laboratory Risk Indicator for Necrotizing Fasciitis (LRINEC) Score for Necrotizing Fasciitis of the Extremities. PLoS One. 2020 Jan 24; 15(1):e0227748. doi: 10.1371/journal.pone.0227748. PMID: 31978094 ; PMCID: PMC6980593.

7. Arif N, Yousfi S, Vinnard C. Deaths from necrotizing fasciitis in the United States, 2003-2013. Epidemiol Infect. 2016; 144(6):1338-44.

8. World Health Organization. (2020). The impact of the COVID-19 pandemic on noncommunicable disease resources and services: results of a rapid assessment. World Health Organization. https://apps.who.int/iris/handle/10665/3341 36. License: CC BY-NC-SA 3.0 IGO

9. Lee YC, Hor LI, Chiu HY, Lee JW, Shieh SJ. Prognostic factor of mortality and its clinical implications in patients with necrotizing fasciitis caused by Vibrio vulnificus. Eur J Clin Microbiol Infect Dis. 2014;33(6):1011-8. doi:10.1007/s10096013-2039-x. Epub 2014 Jan 14.

How to cite this article: Chauhan R, Poddar D, Lohchab P. Necrotizing fasciitis: risk factors and outcomes in the COVID-19 era. International Journal of Research and Review. 2021; 8(8): 645-649. DOI: https://doi.org/10. 52403/ijrr.20210885 